\title{
DISTRIBUCIÓN Y ABUNDANCIA DE MELANOIDES TUBERCULATA (GASTROPODA: THIARIDAE) EN LA RESERVA DE LA BIOSFERA PANTANOS DE CENTLA, TABASCO, MÉXICO.
}

\author{
Natalia C. Albarran-Melze, Luis J. RANGEl-Ruiz \\ y Jaquelina GAMBOA AGUILAR
}

Laboratorio de Malacología, División Académica de Ciencias Biológicas, Universidad Juárez Autónoma de Tabasco. Carretera Villahermosa-Cárdenas Km. 0.5 entronque con Saloya. C. P. 94250 Villahermosa, Tabasco, MÉXICO.makarova2@hotmail.com, ljrangel@msn.com

\begin{abstract}
Albarran-Melze, N. C., L. J. Rangel-Ruiz y J. Gamboa Aguilar. 2009. Distribución y abundancia de Melanoides tuberculata (Gastropoda: Thiaridae) en la Reserva de la Biosfera Pantanos de Centla, Tabasco, México. Acta Zool. Mex. (n. s.) 25(1):93-104.

RESUMEN. Se estudió la distribución y abundancia de Melanoides tuberculata en la Reserva de la Biosfera Pantanos de Centla. Los muestreos se realizaron en 19 lagunas distribuidas en el interior de la Reserva durante la época de lluvias (septiembre y octubre 2003) y en la época de estiaje (abril y mayo 2004). Para conocer el patrón de distribución espacial se utilizaron índices de agregación: Índice de Morisita (Iס) y Parámetro $k$. Se encontró la presencia de esta especie en 18 de las lagunas muestreadas. Se recolectaron 13,597 individuos. La mayor abundancia se presentó en la época de lluvias (8,364 individuos). Las poblaciones en cada laguna presentaron un patrón de distribución espacial agregado. Durante la época de lluvias se encontró una correlación inversa entre la abundancia y demanda química de oxígeno y directa con nitratos, y en la de estiaje inversa entre la abundancia y salinidad y directa con nitratos, nitritos y potencial de hidrógeno.
\end{abstract}

Palabras Clave: Melanoides tuberculata, Thiaridae, Gastropoda, lagunas, distribución espacial, abundancia.

Albarran-Melze, N. C., L. J. Rangel-Ruiz \& J. Gamboa Aguilar 2009. Distribution and abundance of Melanoides tuberculata (Gastropoda: Thiaridae) in the Biosphere reserve of Pantanos de Centla, Tabasco, Mexico. Acta Zool. Mex. (n. s.) 25(1):93-104

ABSTRACT. We studied the distribution and abundance of Melanoides tuberculata in the Centla Swamps Biosphere Reserve. We sampled 19 lagoons, during the rainy (September - October 2003) dry (April - May 2004) seasons. We used the variance: Morisita's Index (Iס) and the $k$ parameter to estimate the distribution pattern space aggregation index. We registered the species 18 lagoons. A total of 13,597 individuals were collected, the greater abundance appeared during the rainy season (8,364 individuals). All populations presented an aggregated spatial distribution pattern. Abundance was inversely correlated with chemical oxygen demand and directly correlated with nitrates during the rainy season. During the dry season, abundance was inversely correlated with salinity and directly with nitrates, nitrites and $\mathrm{pH}$.

Key words: Melanoides tuberculata Thiaridae, Gastropoda, lagoons spatial distribution, abundance. 
Albarran-Melze et al. Melanoides tuberculata en la Reserva de la Biósfera Pantanos de Centla

\section{INTRODUCCIÓN}

Melanoides tuberculata (Müller 1774) es un gasterópodo prosobranquio dulceacuícola que presenta una amplia distribución geográfica en los cuerpos de agua del país, ya que tiene la habilidad de invadir nuevos hábitats. El primer registro en México fue realizado por Abbott en 1973 en Veracruz. Tiene gran importancia ecológica por sus efectos sobre las comunidades de gasterópodos, ya que desplaza y amenaza con desaparecer o al menos disminuir las poblaciones de gasterópodos nativos debido a su elevada tasa de reproducción, ya que es una especie partenogenética y ovovivípara, presenta una longevidad de cuatro a cinco años (Pointier \& McCullough 1989). Un solo individuo puede colonizar y formar una nueva población. Se ha mencionado que su dispersión puede ser por arrastre en ríos, por transportación adventicia y principalmente por la actividad del acuarismo (Morrison 1954, Contreras-Arquieta et al. 1995b). Además, es un agente transmisor de enfermedades, ya que actúa como hospedero intermediario de tremátodos como Clonorchis sinensis y Centrocestus formosanus que son parásitos del humano y de peces de importancia comercial respectivamente (Goldsmith \& Heyneman 1995).

Melanoides tuberculata ha sido utilizada con éxito como agente de control biológico contra los hospederos intermediarios de Schistosoma mansoni, los cuales son diferentes especies de planórbidos pertenecientes al género Biomphalaria (Perera et al. 1989 y Pointier et al. 1989).

El único registro en la Reserva de la Biosfera Pantanos de Centla (RBPC) es el de Cruz-Ascencio et al. (2003) quienes registran su presencia en las lagunas San Pedrito, Sauzo y Larga. Sin embargo, no existe ningún estudio de tipo poblacional que aporte información sobre su abundancia e importancia en el interior de la Reserva, la cual es una zona ecológicamente importante para la captación de carbono por la amplia cobertura vegetal.

En la Reserva escurre un tercio del agua dulce de todo el país; es área de tránsito, alimento y anidación de aves migratorias; regulación del microclima; control de inundaciones y de la erosión de las costas; área biogénica importante para el aporte de nutrientes; contiene muestras de comunidades vegetales con gran diversidad de formas de vida; gran riqueza de flora con más de 260 especies; y en fauna cuenta con más de 170 especies de vertebrados. Este ecosistema sirve como filtro de los escurrimientos que se dirigen a las costas, aportando aguas orgánicas y nutrientes que dan vida y alimento a las especies marinas (SEMARNAP 2000).

La información del patrón de distribución espacial de moluscos es utilizada para determinar el plan de muestreo en los programas de monitoreo de poblaciones animales. Para el índice de Morisita (Iס) cuando sus valores son superiores a uno indica distribuciones agregadas. El Parámetro k de la binomial negativa describe el grado de agregación, y valores altos de $\mathrm{k}$ indican un alto grado de agregación (Southwood 1978, Davis 1994). 
Este trabajo tiene como propósito fundamental conocer la distribución espacial y abundancia de $M$. tuberculata, ya que servirá de base junto con otros estudios, para determinar el efecto que ha tenido sobre el resto de la comunidad de moluscos en la RBPC, ya que en países como Cuba (Gutiérrez et al. 1997), Brasil (Guimaraes et al. 2001) y en las islas de Guadalupe y Martinica (Pointier et al. 1993) se ha registrado el desplazamiento de poblaciones de gasterópodos por M. tuberculata.

\section{MATERIAL Y MÉTODOS}

El área de estudio se encuentra ubicada al noreste del estado de Tabasco, en la zona de descarga de la cuenca de los ríos Grijalva y Usumacinta. Ocupa parte de los municipios de Centla, Jonuta y Macuspana. Se ubica entre las coordenadas geográficas $17^{\circ} 57^{\prime} 53^{\prime \prime}$ y $18^{\circ} 39^{\prime} 03^{\prime \prime}$ de latitud norte y $92^{\circ} 06^{\prime} 39^{\prime \prime}$ y $92^{\circ} 47^{\prime} 58^{\prime \prime}$ de longitud oeste (INEGI 1996). En ésta área se encuentran más de 10 tipos de vegetación entre los que sobresalen manglares, tintales y vegetación hidrófita (Romero et al. 2000).

Los muestreos se llevaron a cabo en septiembre y octubre de 2003 y en abril y mayo de 2004, en una red de 19 lagunas: San Pedrito, Punteada, Guanal, San Isidro, Coco, Cometa, Narváez, Viento, Concepción, Larga, Ídolos, Tintal, Landeros, Sargazal, Loncho, Chichicastle, Sauzo, Jobo y Cantemoc. En cada laguna se tomaron cinco muestras de sedimento en distintos puntos distribuidos al azar. La toma de muestras se realizó con la ayuda de una draga Van Veen con capacidad de 5 litros, colocándolas en cubetas. Posteriormente se procesaron, a través de un conjunto de tamices de 10.0, 2.0 y $0.5 \mathrm{~mm}$ de abertura de luz. Las muestras fueron preservadas y fijadas en alcohol al $70 \%$ y cuantificadas en el laboratorio.

Con las medidas descriptivas (medias y varianzas) se calcularon los índices de agregación de la abundancia de cada una de las dos temporadas muestreadas (lluvias y estiaje). Se seleccionaron al Índice de Morisita (Iס) y al Parámetro $k(k)$ debido a que estos índices no son afectados por la densidad media y además porque en el muestreo se mantuvo el mismo número de muestras (Myers 1978, Mollet et al. 1984, Morisita 1962).

Para determinar los rangos de abundancia se tomó el número total de individuos capturados en la muestra y se agruparon en los siguientes intervalos: abundancias bajas de 1 a 100 organismos, medias de 100 a 1000 y abundancias altas de 1000 en adelante.

Los parámetros fisicoquímicos que se tomaron en campo y laboratorio fueron: Temperatura del aire ( $\mathrm{T}$ aire), Temperatura del agua ( $\mathrm{T}$ agua), Salinidad (Sal), Alcalinidad total (AT), Dureza $\left(\mathrm{CaCO}_{3}\right)$, Sólidos suspendidos totales (SST), Potencial hidrógeno $(\mathrm{pH})$, Demanda química de oxígeno (DQO), Demanda bioquímica de oxígeno $\left(\mathrm{DBO}_{5}\right)$, Visibilidad al disco de Secchi (VDS), Profundidad (Prof), Nitratos $\left(\mathrm{NO}_{3}\right)$, Nitritos $\left(\mathrm{NO}_{2}\right)$, Amonio $\left(\mathrm{NH}_{4}\right)$ y Fósforo total (PT). Las 
muestras de agua fueron colectadas y analizadas de acuerdo a los métodos recomendados por APHA (1992). En los resultados primero se señala el valor mínimo, el máximo y el promedio.

Para determinar las diferencias entre las abundancias de cada una de las localidades de estudio y entre las abundancias de las épocas de lluvias y estiaje en la RBPC y debido a la falta de normalidad de los datos, se realizó una prueba de Kruskal-Wallis y Kolmogorov-Smirnov respectivamente. Para la correlación de los factores fisicoquímicos con la abundancia, se utilizó un Análisis de Correlación de Rangos de Spearman.

\section{RESULTADOS}

Melanoides tuberculata se encontró distribuida en 18 de las 19 lagunas muestreadas; de estas lagunas Loncho, Punteada, Landeros, Ídolos, San Pedrito, Coco y Narváez presentan abundancias muy bajas y la laguna el Cometa es la localidad donde la especie estuvo ausente.

Se recolectó un total de 13,592 individuos. Los valores de abundancia promedio mas altos se presentaron en: Guanal (2,083.0 ind.), San Isidro (1,651.5), Sauzo (1013.0) y Cantemoc (888.0) y los mas bajos en: Coco (2.5), Ídolos (3.0), Landeros (4.0), Narváez (6.0) y Punteada (6.0) (Cuadro 1). Al comparar las abundancias de cada localidad tanto de la época de lluvias como la de estiaje, no existieron diferencias significativas entre las dos épocas $(p=0.33$ y $p=0.30$, respectivamente).

Cuadro 1. Abundancia de M. tuberculata en la Reserva de la Biosfera Pantanos de Centla en dos épocas del año.

\begin{tabular}{lccclccc}
\hline Lagunas & Lluvia & Estiaje & Prom & Lagunas & Lluvia & Estiaje & Prom \\
\hline Guanal & 1048 & 3118 & 2083 & Loncho & 12 & 1 & 6.5 \\
San Isidro & 2035 & 1268 & 1651.5 & Sargazal & 12 & 52 & 32 \\
Sauzo & 2011 & 15 & 1013 & Punteada & 11 & 1 & 6 \\
Cantemoc & 1676 & 100 & 888 & Landeros & 7 & 1 & 4 \\
Tintal & 618 & 376 & 497 & Ídolos & 5 & 1 & 3 \\
Jobo & 582 & 15 & 298.5 & San Pedrito & 4 & 11 & 7.5 \\
Chichicastle & 138 & 160 & 149 & Coco & 4 & 1 & 2.5 \\
Larga & 111 & 100 & 105.5 & Narváez & 1 & 11 & 6 \\
Viento & 38 & 7 & 22.5 & Cometa & 0 & 0 & 0 \\
Concepción & 29 & 12 & 20.5 & & & & \\
\hline
\end{tabular}


Considerando solo las lagunas con altas y moderadas abundancias (San Isidro, Sauzo, Cantemoc, Tintal, Jobo, Chichicastle y Larga) las máximas abundancias se presentaron en la temporada de lluvias con excepción del Guanal (Cuadro 1). Sin embargo considerando el total de las abundancias de las 18 localidades no existió diferencia significativa entre la época de lluvias y la de estiaje $(p=0.847)$.

A pesar de que la abundancia total fue mayor en la época de lluvia (8,342 ind.) que en la de estiaje (5,250 ind.), no existieron diferencias estadísticas entre las dos épocas $(\mathrm{p}=0.50)$.

En cuanto a los índices de agregación, para el Índice de Morisita (Iס) los valores variaron de 2.41 en Tintal a 9.77 en Chichicastle en lluvias, y de 2.78 en San Isidro a 6.10 en Tintal en estiaje. Ya que los valores Is fueron significativamente mayores a uno indican una clara distribución agregada. Los valores del Parámetro $k$ variaron de 0.104 en Chichicastle a 0.639 en Tintal en lluvias y de 0.177 en Tintal a 0.568 en Sauzo en estiaje (Cuadro 2), los valores de $k$ indican un alto grado de agrupamiento. Por lo anterior la distribución espacial obtenida en todas las localidades y en las dos épocas del año es agregada.

Cuadro 2. Índices de agregación para la abundancia de M. tuberculata en la Reserva de la Biósfera Pantanos de Centla.

\begin{tabular}{|c|c|c|c|c|}
\hline & \multicolumn{2}{|c|}{ Lluvia } & \multicolumn{2}{|c|}{ Estiaje } \\
\hline & $\begin{array}{c}\text { Índice de Morisita } \\
\text { (I } \partial \text { ) }\end{array}$ & Parámetro $\mathrm{k}(k)$ & Índice de Morisita $\left(\mathrm{I}_{\delta}\right)$ & Parámetro $\mathrm{k}(k)$ \\
\hline Punteada & 4.76 & 0.28 & NA & NA \\
\hline Guanal & 3.94 & 0.31 & 3.26 & 0.40 \\
\hline San I sidro & 2.96 & 0.46 & 2.78 & 0.50 \\
\hline Viento & 2.53 & 0.60 & 6.00 & 0.23 \\
\hline Concepción & 5.36 & 0.21 & 5.15 & 0.24 \\
\hline Larga & 4.21 & 0.28 & 6.05 & 0.18 \\
\hline Tintal & 2.41 & 0.64 & 6.10 & 0.18 \\
\hline Chichicastle & 9.77 & 0.10 & 3.51 & 0.36 \\
\hline Sauzo & NA & NA & 3.00 & 0.57 \\
\hline Jobo & 6.41 & 0.17 & 3.05 & 0.49 \\
\hline Cantemoc & 3.06 & 0.44 & 4.31 & 0.27 \\
\hline
\end{tabular}

La distribución geográfica de las estaciones con altas abundancias dentro de la reserva estuvieron localizadas en dos subcuencas de la Cuenca Río Usumacinta: Subcuenca Río Usumacinta (Guanal, Cantemoc, Sauzo) y Subcuenca Río San Pedro y San Pablo (San Isidro) y las moderadas en dos subcuencas de la Cuenca Río Grijalva-Villahermosa: Subcuenca Río Grijalva (Larga y Tintal) y Subcuenca Río Chilapa (Chichicastle y San Isidro el Jobo) (INEGI 2000), (Fig. 1). 


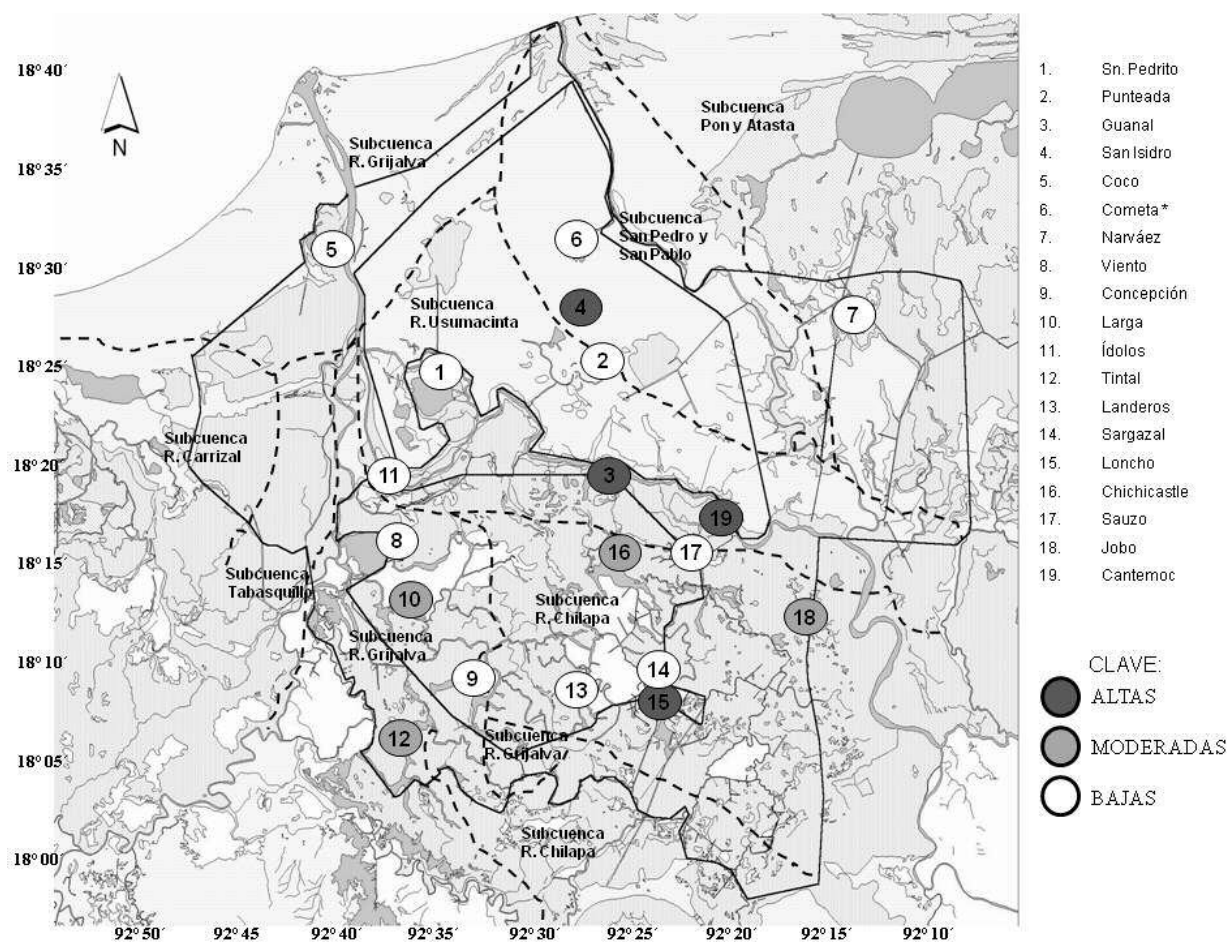

Figura. 1. Abundancia promedio de Melanoides tuberculata en la Reserva de la Biosfera Pantanos de Centla de las dos épocas del año (Bajas 10 - 100, Medias 100 - 1000, Altas > 1000).

Los valores mínimos (MIN), máximos (MAX) y promedio (PROM) de los parámetros fisicoquímicos en la época de lluvias y de estiaje se presentan en el Cuadro 3.

En cuanto a los análisis de correlación, durante la época de lluvias hubo una relación significativa e inversa entre la abundancia y demanda química de oxígeno $(\mathrm{r}=-0.45, \mathrm{p}=0.05)$ y directa con nitratos $(\mathrm{r}=0.57, \mathrm{p}=0.01)$. En la época de estiaje se encontró una relación significativa e inversa entre la abundancia y salinidad $(\mathrm{r}=-0.49, \mathrm{p}=0.04)$ y una directa con nitratos $(\mathrm{r}=0.53, \mathrm{p}=0.03)$, nitritos $(\mathrm{r}=0.57$, $\mathrm{p}=0.02)$ y potencial de hidrógeno $(\mathrm{r}=0.47, \mathrm{p}=0.05)$.

\section{DISCUSIÓN}

La distribución geográfica de M. tuberculata en la RBPC es muy amplia ya que solo estuvo ausente en una de las 19 lagunas muestreadas (Cometa). Esta capacidad de dispersión en la Reserva así como en otras partes del mundo, demuestra su éxito ecológico atribuido a su alto potencial biótico, por ser ovovivípara, partenogenética 
Cuadro 3. Parámetros fisicoquímicos promedio en dos épocas del año en la Reserva de la Biosfera Pantanos de Centla.

\begin{tabular}{|c|c|c|c|c|c|c|}
\hline & \multicolumn{3}{|c|}{ Lluvia } & \multicolumn{3}{|c|}{ Estiaje } \\
\hline & Min & Max & Prom & Min & $\operatorname{Max}$ & Prom \\
\hline $\mathrm{T}$ aire $\left({ }^{\circ} \mathrm{C}\right)$ & 24.0 & 35.0 & 30.58 & 27.0 & 36.0 & 31.22 \\
\hline T agua $\left({ }^{\circ} \mathrm{C}\right)$ & 26.0 & 33.0 & 30.17 & 27.0 & 35.0 & 30.11 \\
\hline $\mathrm{pH}$ & 6.1 & 8.4 & 7.23 & 7.4 & 8.5 & 7.90 \\
\hline $\operatorname{VDS}(\mathrm{m})$ & 0.2 & 1.3 & 0.67 & 0.2 & 0.9 & 0.46 \\
\hline Prof (m) & 0.3 & 2.9 & 1.64 & 0.5 & 3.0 & 1.38 \\
\hline Sal (UPS) & 0.0 & 10.0 & 1.28 & 0.0 & 8.0 & 0.72 \\
\hline $\mathrm{SST}(\mathrm{mg} / \mathrm{L})$ & 5.0 & 26.0 & 13.67 & 5.0 & 153.2 & 31.01 \\
\hline AT $(m g / L)$ & 138.0 & 198.0 & 167.11 & 116.0 & 186.0 & 158.22 \\
\hline $\mathrm{CaCO}_{3}(\mathrm{mg} / \mathrm{L})$ & 74.9 & 351.4 & 124.23 & 216.0 & 2352.0 & 489.84 \\
\hline $\mathrm{DBO}_{5}(\mathrm{mg} / \mathrm{L})$ & 1.0 & 5.7 & 3.26 & 0.8 & 5.3 & 2.90 \\
\hline $\mathrm{DQO}\left(\mathrm{mgO}_{2} / \mathrm{L}\right)$ & 4.2 & 80.0 & 34.28 & 4.0 & 264.0 & 44.64 \\
\hline $\mathrm{NO}_{3}(\mathrm{mg} / \mathrm{L})$ & 0.000 & 0.059 & 0.029 & 0.000 & 0.196 & 0.026 \\
\hline $\mathrm{NO}_{2}(\mathrm{mg} / \mathrm{L})$ & 0.021 & 0.067 & 0.041 & 0.021 & 0.072 & 0.038 \\
\hline $\mathrm{NH}_{4}$ & 0.030 & 0.456 & 0.170 & 0.012 & 0.163 & 0.084 \\
\hline PT $\quad(\mathrm{mg} / \mathrm{L})$ & 0.007 & 0.155 & 0.052 & 0.096 & 0.745 & 0.240 \\
\hline
\end{tabular}

y tener una enorme habilidad de invadir nuevos hábitats, en parte a través del transporte sobre aves acuáticas al adherirse las formas juveniles a las patas o plumas (Contreras-Arquieta et al. 1995b). Además de las anteriores causas, en la RBPC es posible que esta especie se ha distribuido por el arrastre de la vegetación acuática principalmente Eichchornia crassipes (lirio acuático) en la época de lluvias, así como también, por la creación de drenes por actividades petroleras que atraviesan la reserva; o por el trasporte humano debido a actividades pesqueras cuando los caracoles se adhieren a las redes o lanchas de pescadores.

En la República Mexicana M. tuberculata había sido considerada como una especie introducida en los años 60 (Contreras-Arquieta et al. 1995a). Sin embargo los registros de dos especies de Melanoides localizadas en rocas del Cretácico Superior (Maastrichtiano) y Terciario Superior (Paleoceno y Eoceno) que representan ambientes de marismas y pantanos, asociados a un sistema deltáico de los estados de Coahuila y Nuevo León, hace aproximadamente 70 millones de años (Vega et al. 2004), significa que los tiáridos ya formaban parte de nuestra fauna y probablemente el hecho de no haberse registrado anteriormente $M$. tuberculata, en caso de que se presentara en México se debió principalmente a los pocos muestreos realizados o a muestreos poco rigurosos. Sin embargo esto requiere revisarse con mayor profundidad ya que, dadas las características biológicas de la especie, resulta extraño que no se posea alguna referencia, al menos aislada, de su presencia antes de los años 70 . 
En la actualidad $M$. tuberculata se distribuye en el continente americano en Texas, Florida, Arizona (Burch 1982) y California (Taylor 1981) en Estados Unidos de Norteamérica; varias islas del Caribe, Centro y Norte de Sudamérica (Pointier y McCullough, 1989).

La distribución conocida actualmente para la República Mexicana es en los estados de Chiapas (Río Coatancito en Tapachula y Río Huehuetán en Huehuetán), Chihuahua (Río Saúz en Estación Saúz y Ojo de Santo Domingo), Coahuila (Parras de La Fuente, Ojo de Agua en "Parque La Mota", Río Salado en Santa Gertrudis, Río Salado en El Cariño de la Montaña), Durango (Río Peñón Blanco y Balneario en La Concha, Peñón Blanco), Morelos (Las Estacas, Centro Piscícola de Zacatepec y Río Yautepec), Nayarit (Río "sin nombre" en Santa Cruz, San Blas, Río Aticaza en Aticaza, San Blas, Estero La Tobara, la Aguada San Blas y Manantial en Pantanal, Tepic), Nuevo León (Rió La Silla en Guadalupe. Presa Rodrigo Gómez "La Boca”, Santiago, Afluente del Río La Silla), Puebla (Acequia de los Manantiales de San Lorenzo en Tehuacán), Quintana Roo (Cenote $7.8 \mathrm{~km}$ al Oeste de Leona Vicario), Tabasco (Rió Grijalva, Villahermosa) y Tamaulipas (Río Sabinas, del Sistema Río Tamesí, en El Limón) Veracruz (en 42 localidades) (Contreras-Arquieta et al. $1995 \mathrm{a} \mathrm{y} \mathrm{b).}$

Para la Reserva de la Biosfera Pantanos de Centla los únicos registros para $M$. tuberculata eran las lagunas de San Pedrito, Sauzo y Larga (Cruz-Ascencio et al. 2003), por lo que las lagunas de San Isidro, Cantemoc, Guanal, Tintal, Jobo, Chichicastle, Viento, Concepción, Loncho, Sargazal, Punteada, Landeros, Ídolos, Coco y Narváez se presentan como nuevos registros en la región.

En las dos épocas de muestreo las abundancias mas altas se presentaron en la parte este de la reserva y las bajas en la parte sur, observándose que en la parte norte de la Reserva su presencia es casi nula, probablemente debido al incremento de la salinidad en época de estiaje, condiciones que representan una barrera abiótica al buen desarrollo de esta especie. Este comportamiento es contrario al registrado por Roessler et al. (1977) quienes señalan que $M$. tuberculata puede subsistir en estuarios de Florida, E.U., dominados por mangle rojo (Rhizophora mangle) y blanco (Laguncularia racemosa), presentando una densidad de 37,500 ind $/ \mathrm{m}^{2}$. Estas condiciones son iguales a las de la laguna el Cometa, sin embargo en esta localidad no se presentó la especie.

Es probable que la presencia de caracoles con muy bajas abundancias en algunas lagunas se deba a su arrastre por las corrientes durante la época lluvias, ya que las poblaciones no se encuentran establecidas de forma permanente (Cuadro 2).

El patrón de distribución espacial en todas las lagunas en donde se registró a $M$. tuberculata fue agregado, característica afín a la mayoría de las poblaciones de gasterópodos dulceacuícolas y similar al registrado por Iannacone et al. (2003), quienes señalan que esta especie presentó una distribución espacial amontonada (agregada), en las lagunas de Puerto Viejo, en Lima, Perú. 
Además el patrón de distribución agregada se mantuvo en las dos épocas del año (lluvias y estiaje) con valores de I $\delta \mathrm{y} \mathrm{k}$ similares por lo que demuestra que la estacionalidad no afecta el patrón de distribución espacial para esta especie.

Entre los principales parámetros fisicoquímicos que definen la calidad del agua en la RBPC fueron: La temperatura del agua que siempre fue superior a los $21^{\circ} \mathrm{C}$ lo cual es característico de ambientes tropicales (De la Lanza y Lozano 1999), la disminución de la temperatura en la época de lluvias y nortes, fue influenciada principalmente por la variación estacional, la temperatura del agua es uno de los principales parámetros físicos ya que afecta disminuyendo o acelerando la actividad biológica; para el $\mathrm{pH}$ estos valores estuvieron entre 6.1 y 8.5 es decir se trata de aguas ligeramente acidas a ligeramente básicas (De la Lanza 1998), por lo general las aguas naturales (no contaminadas) presentan un rango $\mathrm{pH}$ entre 5 a 9; por la VDS y la profundidad todas las lagunas de la RBPC $(0.3$ a $3.0 \mathrm{~m})$ muestran que se trata de sistemas lénticos someros propios de humedales tropicales y muy propicios para el desarrollo de poblaciones y comunidades de moluscos y en particular gasterópodos como M. tuberculata.

Debido a los valores de salinidad registrados en la Reserva, se presentan tres tipos de sistemas: típicamente dulceacuícolas $(<5.0)$ Guanal, Narváez, Concepción, Ídolos, Tintal, Sargazal, Chichicastle, Sauzo y Jobo; oligohalinos (0.5 a 5.0 ups) San Pedrito, Punteada, San Isidro, Coco, Viento, Larga, Landeros y Loncho; y solamente el Cometa como mesohalino (5 - 18 ups) (Carriker 1967);

Para la temporada de lluvias la dureza en las lagunas de la RBPC son de aguas moderadamente duras ( 75 a $149 \mathrm{mg} / \mathrm{L}$ ) con excepción de San Pedrito y Punteada que se presentan como duras (150 a 300) y solamente Cometa como muy dura (>300) (Sawyer \& McCarty 1978). Estas condiciones de dureza son favorables para el desarrollo de la fauna malacológica, ya que hay evidencias de que aguas blandas afectan el crecimiento y reproducción de estos organismos. Trabajos en laboratorio han demostrado también que aguas extremadamente duras afectan significativamente estos dos aspectos biológicos (Dillon 2000) condición encontrada en el Cometa en la cual no se encontró a $M$. tuberculata.

Los valores de la $\mathrm{DBO}_{5}$ en las dos temporadas y en todas las localidades siempre se mantuvieron dentro de los limites máximos permisibles $(75 \mathrm{mg} / \mathrm{L})$, sin embargo, en el caso de la DQO en la temporada de lluvias y en la de estiaje si se presentaron lagunas que superaron sus limites: San Pedrito, Punteada, Coco, Concepción, Larga y Landeros en la de lluvias y Coco, Cometa, Landeros y Sargazal en estiaje (NOM001-ECOL-1996). Para los nutrientes nitratos, amonio y fósforo total estos presentaron valores inferiores a los niveles máximos permisibles $(5.0,0.5$ y $1.0 \mathrm{mg} / \mathrm{L}$ respectivamente) (Ley federal de derechos, disposiciones aplicables en materia de aguas nacionales 2006).

La abundancia de $M$. tuberculata en la época de lluvia, tuvo una correlación significativa y directa con los nitratos e inversa con la DQO y en la época de estiaje 
una correlación significativa y directa con los nitratos, nitritos y $\mathrm{pH}$ e inversa con la salinidad. Estas relaciones son similares a las encontradas en el Municipio de San Juan y Martínez en Cuba en donde encontraron que la abundancia de Fossaria cubensis y Pseudoseccinea columella son principalmente afectadas por las concentraciones de nitritos y nitratos (Cañete et al. 2004). Lo anterior es importante ya que Stevenson (2000) y Scout et al. (2002) señalan que niveles elevados de nitratos han sido implicados como causa de impactos negativos sobre la flora y fauna en manantiales de la Florida. Sin embargo, en la misma Florida no se encontró ninguna correlación entre la densidad de Pomacea paludosa y concentraciones de nitratos (Norah et al. 2006).

Por sus altas abundancias en algunas lagunas en la RBPC y su capacidad de desplazar a otras poblaciones de gasterópodos con los que convive (ContrerasArquieta 1998) se deberá establecer un monitoreo de estas comunidades a largo plazo.

La presencia de $M$. tuberculata en la RBPC además de ser una especie altamente competitiva es el hospedero intermediario de Centrocestus formosanus parásito de peces de importancia comercial (Goldsmith \& Heyneman 1995), registrado por primera vez en México por López-Jiménez en 1987. C. formosanus ha sido registrado para el estado de Tabasco en peces que fungen como el segundo hospedero intermediario en las localidades del Yumka, El Horizonte y Río Puyacatengo. El rango máximo de infección fue de 3,383 parásitos en un pez Cichlasoma managuense de la localidad de El Horizonte (Scholz \& Salgado-Maldonado 2000), localidades cercanas a la RBPC. Además hay que tomar en cuenta que M. tuberculata es un hospedero potencial de Clonorchis sinensis parásito del humano.

AGRADECIMIENTOS. A PEMEX Exploración y Producción Región Sur por haber financiado el desarrollo del proyecto Evaluación de la Hidrodinámica y Comunidades Bióticas en la Reserva de la Biosfera Pantanos de Centla, del cual forma parte esta investigación. Al laboratorio de análisis fisicoquímicos del Área de Coordinación, Vinculación y Servicios de la División Académica de Ciencias Biológicas de la UJAT, por haber proporcionado los resultados de estos análisis.

\section{LITERATURA CITADA}

Abbott, R.T. 1973. Spread of Melanoides tuberculata. The Nautilus. 87(1): 29.

APHA 1992. Métodos normalizados para el análisis de aguas potables y residuales. 17a. Editorial Díaz de Santos Madrid. 1816 p.

Burch, J.B. 1982. Freshwater snails (Mollusca: Gastropoda) of North America. U. S. Enviromental Protection Agency, Cincinnati, Ohio. 294 p.

Cañete, R., M. Yong, J. Sánchez, L. Wong \& A. Gutiérrez. 2004. Population dynamics of intermediate snail hosts of Fasciola hepatica and some environmental factors in San Juan y Martinez Municipality, Cuba. Memórias do Instituto Oswaldo Cruz, Rio de Janeiro Brazil. 99(3): 257-262.

Carriker, M.R. 1967. In: G.H. Lauff, Publ. 83, American Association for the Advancement of Science, Washington, D.C. 442 p. 
Contreras-Arquieta, A., G. Guajardo-Martínez, \& S. Contreras-Balderas. 1995a. Redescripción de caracol exógeno Thiara (Melanoides) tuberculata (Müller, 1774) (Gastropoda: Thiaridae) y su distribución en México. Publicaciones Biológicas - F.C.B./U.A.N.L., México. 8(1 y 2): 1-16. 1995b. Thiara (Melanoides) tuberculata (Müller, 1774) (Gastropoda: Thiaridae), su probable impacto ecológico en México. Publicaciones Biológicas - F.C.B./U.A.N.L., México. 8(1 y 2): 17-24.

Contreras-Arquieta, A. 1998. New records of the snail Melanoides tuberculata (Müller, 1774) (Gastropoda: Thiaridae) in the Cuatro Cienegas Basin, and its distribution in the state of Coahuila, México. The Southwestern Naturalist. 43(2): 283-286.

Cruz-Ascencio, M., R. Florido, A. Contreras-Arquieta \& A.J. Sánchez. 2003. Registro del caracol exótico Thiara (Melanoides) tuberculata (MÜLLER, 1774) (Gastropoda: Thiaridae) en la Reserva de la Biosfera Pantanos de Centla. Universidad y Ciencia. 19 (38): 101-103.

Davis, P.M. 1994. Statistics for describing populations, pp. 33-54. In: L.P. Pedigo \& G.D. Buntin (Eds.). Handbook of sampling methods for Arthropods in agriculture. CRC Press. London.

De la Lanza, E. 1998. Aspectos fisicoquímicos que determinan la calidad del agua. En: C.L.R. Martínez, (Compilador). Ecología de los Sistemas Acuícolas (1-26). Ed. AGT Editor, S.A. México. 227 p.

De la Lanza, E.G. \& M.H. Lozano. 1999. Comparación fisicoquímica de las Lagunas de Alvarado y Términos. Hidrobiológica 9(1): 15-30.

Dillon, R.T. 2000. The ecology of freshwater molluscs. Cambridge University Press. 509 p.

Goldsmith, R. \& D. Heyneman. 1995. "Parasitología y Medicina Tropical”. Edit. Manual Moderno. México D.F. 599 p.

Guimaraes, C.T., S.C. Pereira \& S.D. Moura. 2001. Possible competitive displacement of planorbids by Melanoides tuberculata in Minas Gerais, Brazil. Memórias do Instituto Oswaldo Cruz, Rio de Janeiro Brazil. 96 (Sppl) 173-176.

Gutiérrez, A.A., P.G. Perera, C.M. Yong \& J.A. Fernández. 1997. Relationships of the prosobranch snails Pomacea paludosa, Tarebia granifera and Melanoides tuberculata with the abiotic environment and freshwater snail diversity in the central region of Cuba. Malacological Review. 30: 39-44.

Iannacone, J., J. Mansilla \& K. Ventura. 2003. Macroinvertebrados en las lagunas de Puerto Viejo en Lima, Perú. Ecología aplicada. 2(1): 116-124 p.

INEGI. 1996. Síntesis cartográfica, Nomenclátor y anexos Cartográficos del Estado de Tabasco. 116 p y mapas.

INEGI. 2000. Cuaderno Estadístico Municipal Centla, Tabasco, Gobierno del Estado H. Ayuntamiento Constitucional. $173 \mathrm{p}$

Ley federal de derechos, disposiciones aplicables en materia de aguas nacionales. Diario Oficial de la Federación. 27 de diciembre 2006.

López-Jiménez, S. 1987. Enfermedades más frecuentes de las carpas cultivadas en México. Acuavisión, Revista Mexicana de Acuacultura. 2(9): 11-13.

Mollet, J.A., J.T. Trumble \& V. Sevacherian. 1984. Comparison of dispersion and regression indices for Tetranichus cinnabarinus (Boisduval) (Acari: Tetranychidae) population in cotton. Environmental Entomology. 13: 1511-1514.

Morisita, M. 1962. I $\delta$ - Index, a measure of dispersion of individuals. Researches Population Ecology. 4: $1-7$

Morrison, J.P.E. 1954. The relationships of old and new world Melanians. Proceedings of the United States National Museum Washington. Mus. 103(3325): 357-394.

Myers, J.H. 1978. Selecting a measure of dispersion of individuals. Environmental Entomology. 7:619-621.

Norah, M.C., P.C. Darby \& Ch.M. Pomory. 2006. Nitrate impact on the Florida apple snail, Pomacea paludosa. Hidrobiologia. 568: 135-143. 
Norma Oficial Mexicana NOM-001-ECOL-1996 que establece los límites máximos permisibles de contaminantes en las descargas de aguas residuales en aguas y bienes nacionales. Diario Oficial de la Federación. 24 junio de 1996.

Perera, G., M. Yong, \& J.R. Ferrer. 1989. Ecology and control of Biomphalaria peregrina in Cuba. Journal of Medical and Applied Malacology. 1: 75-81.

Pointier, J.P., A. Guyard \& A. Mosser. 1989. Biological control of Biomphalaria glabrata and B. straminea by the competitor snail Thiara tuberculata in a transmission site of schistosomiasis in Martinique, French West Indies. Annals of Tropical Medicine and Parasitology. 83(3): 263-269.

Pointier, J.P. \& F. McCullough. 1989. Biological control of the snail hosts of Schistosoma mansoni in the Caribbean area using Thiara spp. Acta Tropical, 46: 147-155.

Pointier, J.P., L. Thaler, A.F. Pernot \& B. Delay. 1993. Invasion of the Martinique Island by the parthenogenetic snail Melanoides tuberculata and the succession of morphs. Acta Ecologica. 14(1): 33-42.

Roessler, M.A., G.L. Beardsley, \& D.C. Tabb. 1977. New records of the introduced snail, Melanoides tuberculata (Mollusca: Thiaridae) in south Florida. Florida Scientist. 40(1); 87-94.

Romero, G.J.C., M.A. García, J.C.A. Bautista \& A. P.H. Pérez. 2000. Caracterización de la Reserva de la Biosfera Pantanos de Centla. Universidad y Ciencia. 15(30): 15-28.

Sawyer C.N. \& McCarty P.L. 1978. Chemistry for Environmental Engineering. 3rd ed. McGraw- Hill. Nueva York, EEUU. 532 pp.

Scholz, T \& G. Salgado-Mandonado. 2000. The introduction and dispersal of Centrocestus formosanus (Nishigori, 1924) (Digenea: Heterophyidae) in Mexico: A review. The American Midland Naturalist. 143: 185-200.

Scott, G. G.H. Means, R.C. Means \& R.P. Meegan, 2002. First magnitude springs of Florida. Open File Report No. 85. Florida Department of Environmental Protection, Tallahassee, Fl.

SEMARNAP-INECOL. 2000. Áreas Naturales Protegidas de México. 64 p.

Southwood, T.R.E. 1978. Ecological Methods with particular reference to the study of Insect populations. ELBS and Chapman and Hall, London. 524 p.

Stevenson, J. 2000. Florida's springs strategies for protection and restoration. Florida Department of Environmental Protection, Tallahassee, Fl.

Taylor, D.W. 1981. Freshwater mollusks of California: a distributional checklist. California Fish and Game. 67(3): 140-163.

Vega, F.J., M del C. Perrilliat \& B. Espinosa. 2004. Registro fósil de moluscos dulceacuícolas en México: aportaciones a la geología histórica. Memorias Octavo Congreso Internacional de Malacología Médica y Aplicada. 73-74 p.

Recibido:27 de junio de 2007

Aceptado: 22 de octubre de 2008 